\title{
Flux Dynamics and the Growth of the Superconducting Phase
}

\author{
Holger Frahm, (a) Salman Ullah, and Alan T. Dorsey \\ Department of Physics, University of Virginia, McCormick Road, Charlottesville, Virginia 22901
}

(Received 29 March 1991)

\begin{abstract}
We present a detailed investigation of the growth of the superconducting phase in a magnetic field. For type-I superconductors, the planar normal-superconductor interface is dynamically unstable. In the nucleation regime this feature leads to interface motion similar to that found in the solid-liquid transition. In the spinodal regime the spinodal growth proceeds by a sequence of phase-slip processes, unique to a system with a complex order parameter. In type-II superconductors, vortex absorption stabilizes a planar interface, despite the negative surface tension.

PACS numbers: 74.55.th, 05.70.Ln, 74.60.-w
\end{abstract}

Consider a rod of superconducting material with critical field $H_{c}$, in a magnetic field $H>H_{c}$ (directed along the axis of the rod), so that the rod is in its normal phase. ${ }^{1}$ If the field is now reduced to below $H_{c}$, the normal phase becomes thermodynamically unstable relative to the superconducting phase. For a controlled growth of the superconducting phase one may embed a small piece of superconducting material (with $H_{c}^{\prime}>H_{c}$ ) in the rod which then acts as a nucleating center due to the proximity effect. As the superconducting phase grows, ${ }^{2}$ two possibilities arise. (i) Positive surface tension of the superconductor-normal interface, i.e., a type-I superconductor, favors a smooth interface, which moves into the normal phase, thereby expelling flux (Meissner effect). This motion leads to dynamical instabilities and the associated pattern-forming processes similar to those found in solid-liquid systems. ${ }^{3}$ In addition, the complex nature of the superconducting order parameter allows the superconducting phase to grow by phase slippage, leading to islands of superconducting phase in a sea of magnetic field. (ii) If the surface tension is negative, i.e., a type-II superconductor, then one would expect the notion of a well-defined interface to break down completely. We find that on small length scales, this breakdown manifests itself through the absorption of vortices (quantized filaments of magnetic flux). Remarkably, on larger length scales, this process stabilizes a planar interface. In this Letter we shall discuss the growth of the superconducting phase in a magnetic field, noting the similarities with the simpler solid-liquid system and emphasizing the qualitative differences. Our results are summarized in Figs. 1-3.

Diffusion model.- Some insight into the dynamics of flux expulsion may be gained from the simplified model of Pippard and Lifshitz for a sharp superconductingnormal interface. ${ }^{4}$ In the normal region the dynamics is given by a diffusion equation for the magnetic field $\mathbf{B}=B(x, y) \hat{\mathbf{z}}$ (in conventional units),

$$
\frac{\partial B}{\partial t}=D_{B} \nabla^{2} B
$$

where the diffusion constant is $D_{B}=c^{2} / 4 \pi \sigma_{n} \approx 10^{-2} \mathrm{~m}^{2}$ $\mathrm{s}^{-1}$ with $\sigma_{n}$ the normal conductivity (in the superconducting region $B=0$ ). The interface is determined by two boundary conditions. Ampère's law and Faraday's law yield for the field $B_{s}$ at the interface

$$
B_{s} v_{n}=-D_{B} \hat{\mathbf{n}} \cdot(\nabla B)_{s},
$$

where $v_{n}$ is the velocity of the interface, $\hat{\mathbf{n}}$ is the normal directed out of the superconducting phase, and the derivative is evaluated at the surface. Local thermodynamic equilibrium at the interface gives

$$
B_{s}=H_{c}\left(1-d_{0} k\right) \text {, }
$$

where $d_{0}=\alpha /\left(H_{c}^{2} / 4 \pi\right)$ is the capillary length with $\alpha$ the surface tension, $k$ is the curvature, and $H_{c}$ is the thermodynamic critical field. ${ }^{5}$ The planar interface is dynamically stable for the normal phase moving into the superconducting phase. ${ }^{4}$ However, when the superconducting phase grows into the normal phase, the interface is dynamically unstable to long-wavelength perturbations, ${ }^{6}$ short-wavelength perturbations being stabilized by the surface tension. ${ }^{7}$ On this level the problem of the growth of the superconducting phase in a magnetic field resembles the familiar diffusion-equation description of the solid phase growing into a supercooled melt, the magnetic field playing the role of the temperature. ${ }^{3}$

Just as in the solid-liquid system, in a type-I superconductor there are two distinct dynamical regimes: (a) In the spinodal regime, $0<H_{e}<H_{c 2}$, where $H_{c 2}$ is the spinodal field (and is equal to the upper critical field for a type-II superconductor) and $H_{e}$ is the external field, there is no free-energy barrier to nucleation of the superconducting phase and, therefore, arbitrarily small seeds can grow. (b) In the nucleation regime, $H_{c 2}<H_{e}<H_{c}$, only seeds of radius larger than a given critical radius can grow; smaller seeds collapse. Unfortunately, the sharp-interface model is unable to describe type-I superconductors in the spinodal regime, or in the nucleation regime with $H_{e}$ close to $H_{c}$, because it neglects the order parameter entirely. In addition, the surface tension is negative for a type-II superconductor, a situation which has no analog in the solid-liquid system, and for which 
this model fails. Thus we shall now resort to the more general Ginzburg-Landau description.

Ginzburg-Landau model.-To model nonequilibrium phenomena we use the time-dependent Ginzburg-Landau (TDGL) equations for the complex order parameter $\psi(\mathbf{x}, t)$ and the magnetic vector potential $\mathbf{A}(\mathbf{x}, t):^{8,9}$

$$
\frac{\partial}{\partial t} \psi(\mathbf{x}, t)=-\frac{\delta F}{\delta \psi^{*}(\mathbf{x}, t)},
$$

where the dimensionless gauge-invariant free-energy functional is 10,11

$$
\begin{aligned}
F=\int d^{3} x\{ & -|\psi|^{2}+\frac{1}{2}|\psi|^{4} \\
& \left.+\left|\left[(i \kappa)^{-1} \nabla-\mathbf{A}\right] \psi\right|^{2}+(\boldsymbol{\nabla} \times \mathbf{A})^{2}\right\}
\end{aligned}
$$

The Ginzburg-Landau parameter $\kappa=\lambda(T) / \xi(T)$, where $\xi(T)$ is the coherence length and $\lambda(T)$ is the penetration length. ${ }^{12}$ Consideration of the surface energy of a planar interface shows that for $\kappa<1 / \sqrt{2}(>1 / \sqrt{2})$ we have a type-I (-II) superconductor. ${ }^{10}$ Ampère's law (neglecting displacement currents) leads to the equation of motion for the gauge field: $\boldsymbol{\nabla} \times(\boldsymbol{\nabla} \times \mathbf{A})=(4 \pi / c) \mathbf{J}$, where the total current $\mathbf{J}=\mathbf{J}_{s}+\mathbf{J}_{n}$. In dimensionless units, the supercurrent is $\mathbf{J}_{s}=(1 / \kappa) \operatorname{Im}\left(\psi^{*} \nabla \psi\right)-\mathbf{A}|\psi|^{2}$ and the normal current $\mathbf{J}_{n}=\Sigma \mathbf{E}$ (Ohm's law) with $\mathbf{E}=-\partial \mathbf{A} / \partial t$. Here $\Sigma$ is the dimensionless conductivity: In the Drude model, $\Sigma \approx k_{B} T_{c} \tau / \hbar$, where $T_{c}$ is the critical temperature and $\tau$ is the Drude relaxation time for the normal metal. Thus,

$$
\Sigma \frac{\partial \mathbf{A}}{\partial t}=\mathbf{J}_{s}-\nabla \times(\boldsymbol{\nabla} \times \mathbf{A})
$$

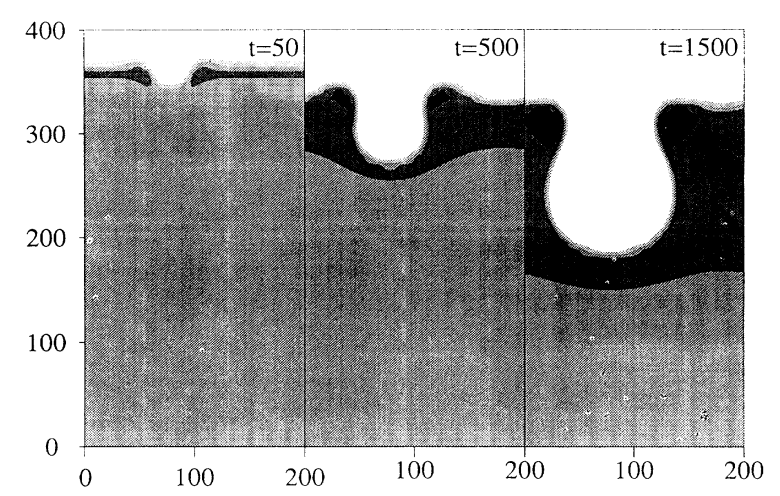

FIG. 1. The magnetic field $B$ in the $x-y$ plane, for a type-I superconductor in the nucleation regime, from Eqs. (4) and (6). The lengths are in units of the penetration depth $\lambda(T)$, time is in units of the order-parameter relaxation time $\tau_{R}$, the magnetic field is in units of $\sqrt{2} H_{c}$, and the grey scale ranges from white $(B=0)$ to black $\left(B \approx H_{c}\right)$. The external magnetic field is $H_{e}=0.4, \kappa=0.3$, and $\Sigma=0.1$ (see text). We begin with a wedge-shaped perturbation on a planar superconductingnormal interface. The interface velocity is proportional to the field gradient, and the magnetic field is large in regions of negative curvature. These two features are expected on the basis of the simple diffusion model.
In addition, we need to specify boundary conditions at the sample-vacuum interface. (a) For the magnetic field $\mathbf{B}=\boldsymbol{\nabla} \times \mathbf{A}$, we require that the tangential component be continuous. (b) For the order parameter, the natural boundary condition is $\mathbf{J} \cdot \hat{\mathbf{n}}=0$, where $\mathbf{J}$ is the total current. ${ }^{13}$ This condition is difficult to impose numerically, so we adopt one of two simpler possibilities: zero supercurrent flowing into the vacuum or zero order parameter at the boundary. The choice of boundary conditions on the order parameter has no effect on the growth provided the superconducting region is several coherence lengths away from the boundary. Finally, in order to reduce boundary effects we impose periodic boundary conditions in the $x$ direction. In our computations we restrict spatial variations to the $x-y$ plane, thereby envisaging a superconductor of infinite extent in the $z$ direction. ${ }^{14}$ It is essential to put the gauge field $\mathbf{A}$ on the links of the computational lattice, ${ }^{15}$ which is achieved by introducing the link variables $U_{\mathrm{x}}^{\mu}=\exp \left[-i \kappa a A^{\mu}(\mathrm{x})\right]$, where $a$ is the lattice constant and $\mu=x, y$ (we suppress the time argument). ${ }^{16}$ In the free energy we make the replacements

$$
\begin{aligned}
& {\left[(i \kappa)^{-1} \partial_{\mu}-A_{\mu}(\mathbf{x})\right] \psi(\mathbf{x})} \\
& \rightarrow(i \kappa a)^{-1}\left[U_{\mathbf{x}}^{\mu} \psi(\mathbf{x}+a \hat{\mu})-\psi(\mathbf{x})\right], \\
& a^{2}[\nabla \times \mathbf{A}(\mathbf{x})]_{z} \\
& \rightarrow i \kappa^{-1}\left[U_{\mathbf{x}}^{x} U_{\mathbf{x}+a \hat{\mathbf{x}}}^{y}\left(U_{\mathbf{x}+a \hat{\mathbf{y}}}^{x}\right)^{-1}\left(U_{\mathbf{x}}^{y}\right)^{-1}-1\right],
\end{aligned}
$$

and solve the corresponding equations of motion using a simple iterative scheme on lattices of size ranging from $100 \times 100$ to $200 \times 200$ sites.

Type-I superconductors. - The growth of the superconducting phase leads to flux buildup in front of the interface until the magnetic field reaches the critical value $H_{c}$. Further growth of the superconducting phase is determined largely by the (diffusive) expulsion of flux from the region in front of the interface. As mentioned above (and verified explicitly by our computations) there are two distinct regions in the $H-\kappa$ plane for type-I superconductors. ${ }^{17}$

Nucleation regime. - We find that the interface moves diffusively only. ${ }^{18}$ Localized perturbations in a planar interface of size less than the critical size $\rho_{c}$ collapse, ${ }^{19}$ while perturbations of size greater than $\rho_{c}$ grow faster than the planar front. In regions of negative curvature, growth of the order parameter is further inhibited by the magnetic field becoming larger than $H_{c}$. The result is a bulbous superconducting front; see Fig. $1 .{ }^{20}$ Our numerical results for the TDGL equations seem to confirm the qualitative predictions of the simple diffusion model ${ }^{21}$ provided $H_{e}$ is sufficiently less than $H_{c}$. Eventually, all the flux is expelled and the superconductor attains the Meissner phase. The analogy to the solid-liquid system suggests the possibility of dendritic growth of the superconductor, i.e., needlelike solutions growing with constant velocity, in the presence of anisotropy. In addition 
to the lattice anisotropy, we introduce an explicit $m$-fold anisotropy in the order-parameter relaxation rate. The tip radius $\rho_{\text {tip }}$ of the dendrite may be estimated from the diffusion model. ${ }^{3}$ On the lattice sizes accessible to us, the lattice anisotropy alone has little effect on the growth characteristics, which suggests that $\rho_{\text {tip }}$ is very large. For the modified model with maximal anisotropy we obtain a minimal tip radius of $\rho_{\text {tip }} \approx 70$ penetration lengths, which is still a large fraction of our largest lattice. Nevertheless, given that the diffusion model seems to be qualitatively correct, we expect anisotropy to result in dendritic patterns in the nucleation regime.

Spinodal regime.-At small times, the superconducting phase grows in the same manner as in the nucleation regime, i.e., diffusively. However, in the spinodal regime an interesting possibility arises. The order parameter can leak through the flux wall giving rise to a new seed on the other side-see Fig. 2. This process is unique to a system with a complex order parameter coupled to a gauge field. If the amplitude of $\psi$ is small over a given distance, then it is possible for the phase $\phi$ of the order parameter to change by $2 \pi$ over the same distance. This possibility-a phase slip-reduces the gradient term in the free energy (5) $(\mathbf{A} \rightarrow \mathbf{A}-\nabla \phi / \kappa)$, allowing the order parameter to grow again. This process repeats itself, leading to growing islands of the superconducting phase, with a web of trapped flux in between where the field attains a value of $H_{c}$. Eventually the enclosed flux moves towards the boundary of the sample and is expelled.

Type-II superconductors.-We begin with a superconducting seed in a uniform magnetic field. In the fluxlattice regime $H_{e}>H_{c 1}$, the interface moves with constant velocity until the superconductor becomes unstable to vortex absorption. At this point, the interface slows

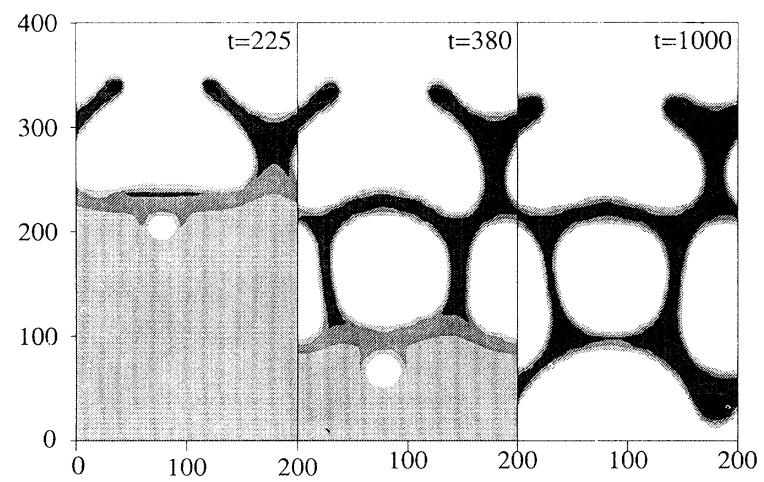

FIG. 2. The magnetic field for a type-I superconductor in the spinodal regime. The initial conditions and relevant parameters are the same as in Fig. 1 except that $\boldsymbol{H}_{e}=0.2$. There is no lower critical size for seed nucleation so that the order parameter can leak through the flux wall via the mechanism of phase slippage (see text), resulting in a daughter seed on the other side of the wall. The daughter seed continues to grow until once more the field at the interface increases to $H_{c}$, and the process repeats. down significantly and absorbs vortices-see Fig. 3-a unique process for pattern-forming systems. The interface continues to move at a constant velocity until once more it becomes energetically favorable to absorb more vortices. The vortices slowly form domains of a triangular flux lattice, separated by line defects. Including thermal noise in our computations would tend to anneal these defects. The absorption of vortices stabilizes the planar interface on length scales greater than $\xi(T)$ and the characteristic time of this process is set by the order-parameter relaxation time $\tau_{R}$. Hence, the growth rate is insensitive to $\Sigma$. As $H_{e} \rightarrow H_{c 2}$ the growth rate decreases simply because more vortices per unit area have to be absorbed. At $\boldsymbol{H}_{c_{2}}$ the superconducting phase vanishes. For small external fields $H_{e}<H_{c 1}$, vortex absorption occurs due to flux buildup in front of the interface but at long times the vortices are pushed out of the sample, resulting in the expected equilibrium Meissner phase. $^{22}$

We have presented a detailed study of the dynamics of flux expulsion in superconductors in a magnetic field. In the nucleation regime type-I superconductors grow diffusively at short times and may show dendritic growth on larger scales; in the spinodal regime leakage of the order parameter occurs, resulting in flux trapping at intermediate times. Type-II superconductors are altogether more exotic: The absorption of quantized vortices stabilizes the superconductor-normal interface. We hope that these results will encourage experimentalists to investigate flux dynamics and pattern formation in superconductors.

We are grateful to N. Goldenfeld for helpful discussions and for sending us unpublished results. We thank Hank Thacker for detailed discussions on lattice gauge theory, and D. Huse and C. Lobb for useful conversations. Using similar techniques Liu, Mondello, and Gol-

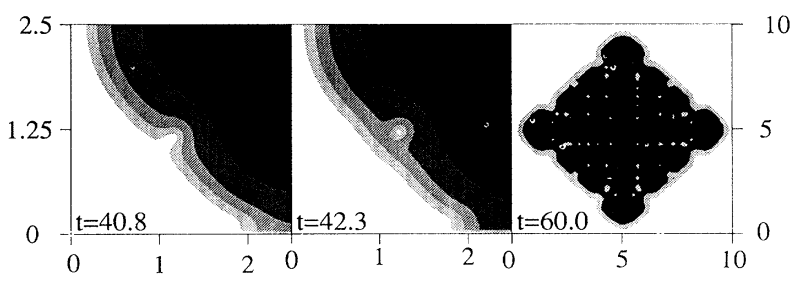

FIG. 3. The amplitude of the order parameter for a type-II superconductor. Here $\kappa=20, H_{e}=1.0$, and $\Sigma=0.1$, and the grey scale ranges from white $(|\psi|=0)$ to black $(|\psi|=1)$; on the scale shown, the magnetic field varies very little from $H_{e}$. We start with a superconducting seed at the center of the lattice in a uniform magnetic field. It grows until vortex absorption becomes energetically favorable. The first two panels show the absorption of a single vortex at the interface: The vortex enters at a corner of the computational lattice. The third panel shows (i) the effect of the fourfold symmetry of the lattice on the large-scale shape of the superconducting region, and (ii) the stability [on lengths larger than $\lambda(T)$ ] of a planar interface. 
denfeld ${ }^{23}$ have recently studied the coarsening problem in this system. This work was supported by NSF Grants No. DMR 88-10541 and No. DMR 89-14051, and Virginia Center of Innovative Technology Grant No. CIT SUP-89-004.

(a) Present address: Institut für Theoretische Physik, Universität Hannover, 3000 Hannover 1, Federal Republic of Germany.

'Superconductivity, edited by R. D. Parks (Marcel Dekker, New York, 1969), Vols. 1 and 2.

${ }^{2}$ Magneto-optics [J. D. Livingstone and W. DeSorbo, in $S u$ perconductivity (Ref. 1), p. 1235] and electron holography [T. Matsuda, A. Fukuhara, T. Yoshida, S. Hasegawa, A. Tonumura, and Q. Ru, Phys. Rev. Lett. 66, 457 (1991)] are possible experimental techniques for studying flux dynamics in superconductors.

${ }^{3} \mathrm{~J}$. S. Langer, in Chance and Matter, Proceedings of the Les Houches Summer School, Session 46, edited by J. Souletie, J. Vannimenus, and R. Stora (North-Holland, Amsterdam, 1987), p. 629.

${ }^{4}$ A. B. Pippard, Philos. Mag. 41, 243 (1950); I. M. Lifshitz, Zh. Eksp. Teor. Fiz. 20, 834 (1950).

${ }^{5}$ C. G. Kuper, Philos. Mag. 42, 961 (1951). We thank N. Goldenfeld for correcting a factor-of- 2 error in a preliminary version of the manuscript.

${ }^{6}$ In the liquid-solid system, this instability is known as the Mullins-Sekerka instability: W. W. Mullins and R. F. Sekerka, J. Appl. Phys. 35, 444 (1964).

${ }^{7}$ We have assumed that the superconductor is translationally invariant in the $z$ direction. We do not expect further dynamical instabilities for perturbations along the $z$ direction because the concomitant bending of the magnetic-field lines is energetically unfavorable: L. Landau, E. Lifshitz, and L. Pitaevksii, Electrodynamics of Continuous Media (Pergamon, Oxford, 1984), 2nd ed., Sec. 56. This question will be discussed in a future publication.

${ }^{8}$ A. Schmid, Phys. Kondens. Mater. 5, 302 (1966); E. Abrahams and T. Tsuneto, Phys. Rev. 152, 416 (1966).

${ }^{9}$ The choice of the temporal gauge-scalar potential $\Phi=0-$ is very convenient since $\Phi$ is not generated by the equations of motion.
${ }^{10}$ A. L. Fetter and P. C. Hohenberg, in Superconductivity (Ref. 1), p. 902.

${ }^{11}$ Time is measured in units of the order-parameter relaxation time $\tau_{R} \approx 10^{-11}\left(1-T / T_{c}\right)^{-1} \mathrm{~s}$ (see Ref. 8). Lengths are measured in units of the penetration length $\lambda(T)=\lambda_{0}[1$ $\left.-\left(T / T_{c}\right)\right]^{-1 / 2}$, where $\lambda_{0} \approx 10 \mathrm{~nm}$, the order parameter has been scaled by its bulk value, and the vector potential is in units of $\sqrt{2} H_{c} \lambda(T)$, where $H_{c}$ is the thermodynamic critical field (see Ref. 10).

${ }^{12} \psi$ and the magnetic field $\mathbf{B}=\boldsymbol{\nabla} \times \mathbf{A}$ vary on length scales of $\xi(T)$ and $\lambda(T)$, respectively.

${ }^{13}$ We emphasize that the interface conditions for the Pippard-Lifshitz model, Eqs. (2) and (3), have no analog in the TDGL theory because of TDGL equations alone provide the self-consistent description of the coupling between the order parameter and the vector potential.

${ }^{14} \mathrm{We}$ do not consider the effect of demagnetization factors. These effects lead to interesting magnetic-field patterns in type-I superconductors: R. P. Heubener, Magnetic Flux Structures in Superconductors (Springer-Verlag, Berlin, 1979). This problem is intrinsically three dimensional.

${ }^{15} \mathrm{~N}$. Goldenfeld (private communication).

${ }^{16}$ K. J. M. Moriarty, E. Myers, and C. Rebbi, Comput. Phys. Commun. 54, 272 (1989); H. B. Thacker (private communication). Note that the argument of the exponential must be less than $\pi$, which limits the maximum value of the lattice constant $a$.

${ }^{17} \mathrm{We}$ do not discuss the problem of surface nucleation: J. P. Burger and D. Saint-James, in Superconductivity (Ref. 1), p. 977.

${ }^{18}$ In zero field the interface moves at a constant velocity $v_{0}=2 / \kappa$ : D. G. Aronson and H. F. Weinberger, Adv. Math. 30, 33 (1978).

${ }^{19}$ The critical radius $\rho_{c}$ is obtained by minimizing the free energy of condensation of a drop: $\rho_{c} \sim 1 /\left[1-\left(B / H_{c}\right)^{2}\right]$.

${ }^{20}$ At longer times our results indicate a possible tip-splitting instability - see D. A. Kessler, J. Koplik, and H. Levine, Adv. Phys. 37, 255 (1988).

${ }^{2 \mathrm{I}}$ The equation for the gauge field (6) reduces to the diffusion equation (1) if $\psi=0$.

${ }^{22}$ Y. Enomoto and R. Kato, J. Phys. Condens. Matter 3, 375 (1991).

${ }^{23}$ F. Liu, M. Mondello, and N. Goldenfeld, following Letter, Phys. Rev. Lett. 66, 3071 (1991). 


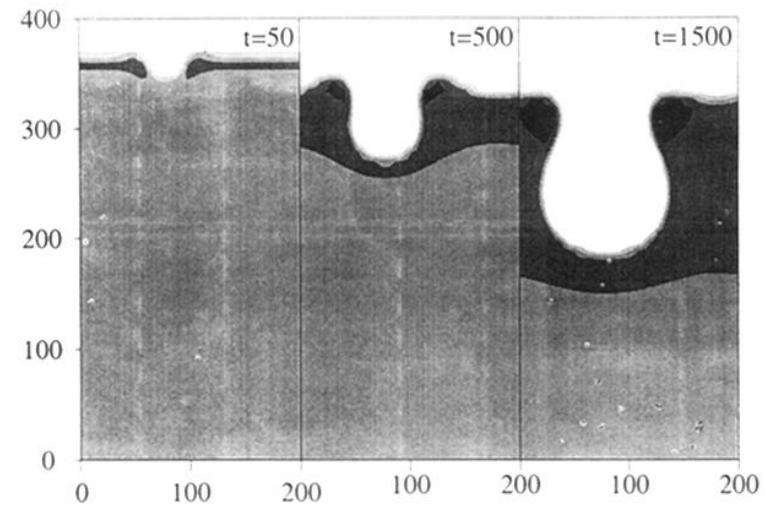

FIG. 1. The magnetic field $B$ in the $x-y$ plane, for a type-I superconductor in the nucleation regime, from Eqs. (4) and (6). The lengths are in units of the penetration depth $\lambda(T)$, time is in units of the order-parameter relaxation time $\tau_{R}$, the magnetic field is in units of $\sqrt{2} H_{c}$, and the grey scale ranges from white $(B=0)$ to black $\left(B \approx H_{c}\right)$. The external magnetic field is $H_{e}=0.4, \kappa=0.3$, and $\Sigma=0.1$ (see text). We begin with a wedge-shaped perturbation on a planar superconductingnormal interface. The interface velocity is proportional to the field gradient, and the magnetic field is large in regions of negative curvature. These two features are expected on the basis of the simple diffusion model. 


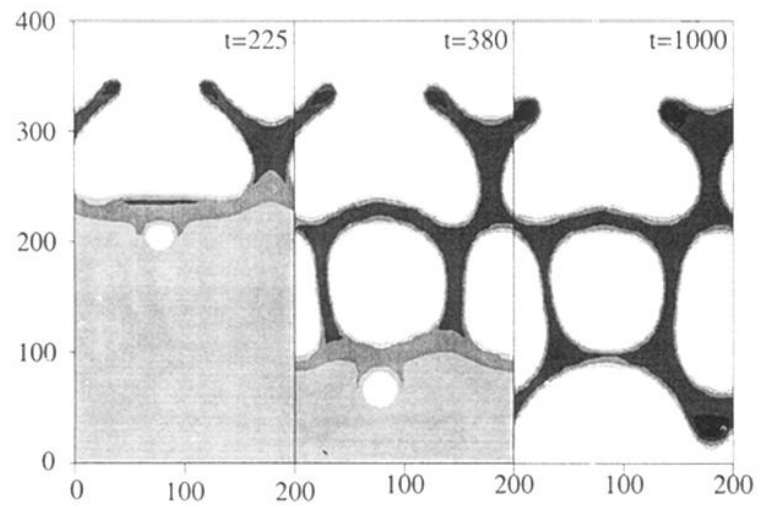

FIG. 2. The magnetic field for a type-I superconductor in the spinodal regime. The initial conditions and relevant parameters are the same as in Fig. 1 except that $H_{e}=0.2$. There is no lower critical size for seed nucleation so that the order parameter can leak through the flux wall via the mechanism of phase slippage (see text), resulting in a daughter seed on the other side of the wall. The daughter seed continues to grow until once more the field at the interface increases to $H_{c}$, and the process repeats. 


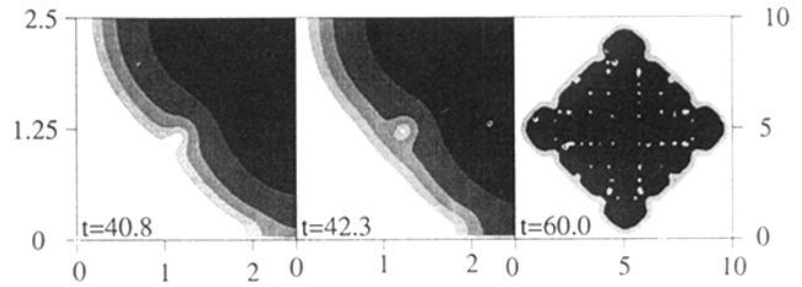

FIG. 3. The amplitude of the order parameter for a type-II superconductor. Here $\kappa=20, H_{e}=1.0$, and $\Sigma=0.1$, and the grey scale ranges from white $(|\psi|=0)$ to black $(|\psi|=1)$; on the scale shown, the magnetic field varies very little from $H_{e}$. We start with a superconducting seed at the center of the lattice in a uniform magnetic field. It grows until vortex absorption becomes energetically favorable. The first two panels show the absorption of a single vortex at the interface: The vortex enters at a corner of the computational lattice. The third panel shows (i) the effect of the fourfold symmetry of the lattice on the large-scale shape of the superconducting region, and (ii) the stability [on lengths larger than $\lambda(T)$ ] of a planar interface. 